\title{
THE GEOMETRY OF THE HYPERELLIPTIC INVOLUTION IN GENUS TWO
}

\author{
ANDREW HAAS AND PERRY SUSSKIND
}

(Communicated by Irwin Kra)

\begin{abstract}
The hyperelliptic involution of a genus two Riemann surface leaves invariant every simple closed geodesic on the surface. This property is not possessed by any other automorphism of a compact Riemann surface and is related to the effectiveness of the action of the mapping class group of a surface on Teichmüller space.
\end{abstract}

A hyperelliptic involution of a genus $p$ Riemann surface is an order two conformal automorphism of the surface that fixes $2 p+2$ points. When such an automorphism of a surface exists it is known to be unique and the surface is said to be hyperelliptic. Every surface of genus two is hyperelliptic.

Geodesics are defined in the complete Riemannian metric of constant curvature -1 (hyperbolic metric) which is uniquely determined by the conformal equivalence class of metrics for the given Riemann surface. A geodesic is simple if it has no self-intersections.

We are happy to thank Bernie Maskit for reading the original draft and suggesting several significant improvements.

To begin we will prove the following

Theorem 1. Let $J$ be the hyperelliptic involution of a genus two Riemann surface $M$. Then every simple closed geodesic on $M$ is mapped onto itself by $J$. Furthermore, suppose $\alpha$ is a simple closed geodesic on $M$. If $\alpha$ is a dividing curve then $J$ preserves the orientation of $\alpha$ and if $\alpha$ does not divide $M$ then $J$ reverses the orientation of $\alpha$.

The idea of the proof is to cut up $M$, induce involutions on the pieces, and then glue the pieces back together. For the sake of completeness we will reproduce some arguments from [3].

Let $S$ be a domain in the unit disc from which two smaller discs have been removed. $S$ has a hyperbolic metric with respect to which there exist precisely

Received by the editors July 27, 1987 and, in revised form, February 24, 1988.

1980 Mathematics Subject Classification (1985 Revision). Primary 30F10, 30F35.

The first author was partially supported by a NSF grant. 
three simple closed geodesics $\alpha, \beta$ and $\gamma$ each homotopic to one of the boundary circles. Orient the geodesics in the clockwise direction. Let $l(c)$ denote the length of the curve $c$ in the hyperbolic metric.

Lemma 1. If $l(\alpha)=l(\beta)$ then there is a conformal involution $j: S \rightarrow S$ that interchanges $\alpha$ and $\beta$ and keeps invariant $\gamma$.

Proof. Let $\Gamma_{s}$ be a torsion-free Fuchsian group that acts on the upper half-plane $\mathbf{H}$ and represents the surface $S$. Choose a pair of free generators $A$ and $B$ for $\Gamma_{s}$ that cover the geodesics $\alpha$ and $\beta$, respectively. Suppose $A$ and $B$ translate along their fixed axes $\widetilde{\alpha}$ and $\widetilde{\beta}$ in the direction of the orientation lifted from $\alpha$ and $\beta$. Let $j_{0}$ be the elliptic Möbius transformation of order two with fixed points $\xi$ and $\bar{\xi}$, where $\xi$ is the midpoint of the common perpendicular $\sigma$ to $\widetilde{\alpha}$ and $\widetilde{\beta}$. By considering the action of $j_{0}$ on $\sigma$ we see that $j_{0}$ interchanges $\widetilde{\alpha}$ and $\widetilde{\beta}$ with orientations preserved. Thus $j_{0}$ conjugates $A$ to $B$ and $B$ to $A$ and therefore $j_{0}$ normalizes $\Gamma_{s}$. Let $j$ be the involution of $S$ induced by $j_{0}$. Clearly, $j$ interchanges $\alpha$ and $\beta$. Since $j$ permutes the three simple closed geodesics, $j$ must keep $\gamma$ invariant.

Two remarks are in order. First, since $S$ is a plane domain of finite connectivity, the involution $j$ must extend to a Möbius transformation. It follows that $j$ has a single fixed point on $S$. Second, notice that the geodesic $\gamma$ is mapped onto itself by $j$ with orientation preserved. Consequently if $g$ is a primitive transformation in $\Gamma$ covering $\gamma$ then there must be a transformation $h \in \Gamma^{*}$, where $\Gamma^{*}$ is $\Gamma$ extended by $j_{0}$, with $h^{2}=g$. It follows that $h$ also projects to the involution $j$.

An easy consequence of the lemma is the following

Proposition 1. Let $T$ be a Riemann surface of genus one from which a disc has been removed and let $\Gamma$ be a torsion-free Fuchsian group representing $T$. Let $\gamma$ be the simple closed geodesic parallel to the boundary of $T$. Then $T$ admits a conformal involution $j$ with three fixed points. Given any primitive $g \in \Gamma$ covering $\gamma$ there is a lift $j_{0}$ of $j$ with $j_{0}^{2}=g$. Furthermore, if $\alpha$ is any simple closed geodesic on $T$ different from $\gamma$ then $j$ maps $\alpha$ onto itself with orientation reversed.

A complete proof of the proposition can be found in [3]. We will provide a sketch. Cut $T$ open along $\alpha$ to get a sphere with three holes. The sphere is then isometrically embedded in a homeomorphic surface $S$ for which the metric is complete. From Lemma 1 we then infer the existence of an involution that interchanges the two geodesics on $S$ corresponding to the two copies of $\alpha$. This induces an involution $j$ of $T$ by regluing the two copies of $\alpha$. Thus $\alpha$ is mapped onto itself with orientation reversed.

As a consequence of the first remark, $j$ has a single fixed point on the surface $T \backslash\{\alpha\}$. Since the involution maps $\alpha$ onto itself with orientation reversed, $j$ must also have two fix points on $\alpha$. This accounts for the three fixed points of $j$. 
By the second remark the involution is induced by the square root of a primitive boundary transformation and so it is independent of the choice of the simple closed dividing geodesic $\alpha$.

Proof of Theorem 1. Let $G$ be a torsion-free Fuchsian group representing the genus-two Riemann surface $M$. Let $\gamma$ be an oriented simple closed dividing geodesic on $M$. The complement of $\gamma$ on $M$ is the disjoint union of two surfaces $T_{1}$ and $T_{2}$, both surfaces of genus one with a disc removed. Say $T_{1}$ is to the left of $\gamma$.

Let $C$ be the preimage of $\gamma$ under the universal covering map and let $\tilde{\gamma}$ be a connected component of $C$. Denote by $\widetilde{T}_{1}$ and $\widetilde{T}_{2}$ the connected components of $\mathbf{H} \backslash C$ which are adjacent along $\tilde{\gamma}$, with $\widetilde{T}_{1}$ to the left of $\tilde{\gamma}$ (with the orientation lifted from $\gamma$ ). $\widetilde{T}_{1}$ and $\widetilde{T}_{2}$ are stabilized by subgroups $\Gamma_{1}$ and $\Gamma_{2}$ of $G$. Thus the quotients $H / \Gamma_{1}$ and $H / \Gamma_{2}$ are tori with holes in which the surfaces $T_{1}=\widetilde{T}_{1} / \Gamma_{1}$ and $T_{2}=\widetilde{T}_{2} / \Gamma_{2}$ embed isometrically.

$G$ is easily seen to be generated by the transformations in the subgroups $\Gamma_{1}$ and $\Gamma_{2}$; in fact $G$ is the free product of $\Gamma_{1}$ and $\Gamma_{2}$ with amalgamation along the stabilizer of $\tilde{\gamma}$. Let $g$ be a generator for the stabilizer of $\tilde{\gamma}$ in $G$, and let $h$ be a transformation with $h^{2}=g$.

Since $g$ covers the boundary geodesic on $T_{1}$ and $T_{2}$ we may infer from the proposition that $h$ normalizes $T_{1}$ and $T_{2}$ and therefore that it also normalizes $G$. Thus $h$ projects to a conformal involution $J$ of $M$ which may be viewed as the result of gluing together the induced involutions on $T_{1}$ and $T_{2}$. It also follows that $\gamma$ is mapped onto itself with orientation preserved.

The involution $J$ has three fixed points on each of the subsurfaces $T_{1}$ and $T_{2}$. It is therefore the unique hyperelliptic involution of $M$. A similar construction starting with a different choice of initial dividing geodesic $\gamma$ will produce the same hyperelliptic involution $J$. So we see that $J$ maps every simple closed dividing geodesic onto itself with orientation preserved.

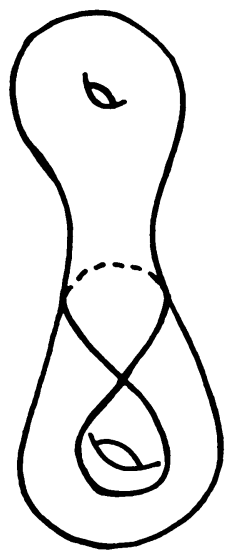

FIGURE 1 
If $\alpha$ is a simple closed nondividing geodesic on $M$ then we can choose a simple closed dividing geodesic $\gamma$ disjoint from $\alpha$ and construct $J$ as above. Since $\alpha$ lies on one of the one-holed tori $T$ in the complement of $\gamma$, the proposition is applicable and we conclude the $\alpha$ is mapped onto itself with its orientation reversed.

One easily constructs examples of nonsimple geodesics on $M$ that are not kept invariant by the involution. See, for example, Figure 1.

Theorem 2. Let $N$ be a compact surface of genus $p>1$ and let $f: N \rightarrow N$ be an orientation-preserving homeomorphism. Suppose that for every simple closed curve $\alpha$ on $N, f(\alpha)$ is freely homotopic to either $\alpha$ or $-\alpha$. Then either $f$ is homotopic to the identity or $N$ is a surface of genus two, and for any complex analytic structure on $N, f$ is homotopic to the hyperelliptic involution.

The proof will be facilitated by the following

Lemma 2. Let $N$ and $f$ satisfy the hypothesis of Theorem 2. Then either $f$ is homotopic to the identity or there is a complex analytic structure on $N$ with respect to which $f$ is homotopic to a conformal involution.

Proof. It is well known that every homeomorphism of a compact surface is homotopic to a diffeomorphism. We will therefore suppose that $f$ is a diffeomorphism. The composition $g=f \circ f: N \rightarrow N$ has the further property that every simple closed curve $\alpha$ is freely homotopic to its image $g(\alpha)$.

Let $G$ be a torsion-free Fuchsian group that acts on $\mathbf{H}$ and represents the surface $N$. Every lift of $f$ to $\mathbf{H}$ extends to a homeomorphism of $\mathbf{H} \cup \mathbf{R} \cup\{\infty\}$. Choose a simple closed nondividing curve $\gamma$ on $N$ and a lift $\tilde{\gamma}$ of $\gamma$ to $\mathbf{H}$. Let $\tilde{g}: \mathbf{H} \rightarrow \mathbf{H}$ be a lift of $g$ to $\mathbf{H}$. Since $g(\gamma)$ is in the same free homotopy class as $\gamma$ and $\tilde{g}$ conjugates $G$ onto $G$ it follows that the endpoints of $\tilde{g}(\tilde{\gamma})$ in $\mathbf{R} \cup\{\infty\}$ are the same as the endpoints of $A(\tilde{\gamma})$ for some $A \in G$. Then $A^{-1} \circ \tilde{g}$ leaves invariant the endpoints of $\tilde{\gamma}$. Without loss of generality we normalize so that the lift $\tilde{g}$ leaves invariant the endpoints of $\tilde{\gamma}$. Since $g$ preserves orientation on $\gamma, \tilde{g}$ must actually fix the endpoints of $\tilde{\gamma}$. Consequently $\tilde{g}$ commutes with a primitive hyperbolic transformation $A_{\tilde{\gamma}}$ in $G$ leaving $\tilde{\gamma}$ invariant.

Let $\sigma$ be a simple closed geodesic that intersects $\gamma$ in a single point, and let $\tilde{\sigma}$ be a lift of $\sigma$ that intersects $\tilde{\gamma}$. Since $\tilde{g}$ fixes the endpoints of $\tilde{\gamma}$ we get $\tilde{g}(\tilde{\sigma}) \cap \tilde{\gamma} \neq \varnothing$. It follows that for some $n \in \mathbf{Z}, A_{\tilde{\gamma}}^{-n} \circ \tilde{g}$ fixes the endpoints of $\tilde{\sigma}$ and therefore that the map $\hat{g}=A_{\tilde{\gamma}}^{-n} \circ \tilde{g}$ is a lift of $g$ that commutes with both $A_{\hat{\gamma}}$ and $A_{\tilde{\sigma}}$. Consequently $\hat{g}$ fixes the endpoints of the geodesics $\tilde{\gamma}$ and $A_{\tilde{\gamma}}^{k}(\tilde{\sigma})$ for all $k \in \mathbf{Z}$. By considering the action of $\hat{g}$ on endpoints it is easily seen that $\hat{g}$ commutes with every transformation $A_{\tilde{\alpha}}$ in $G$ where $\widetilde{\alpha}$ projects to a simple closed geodesic on $N$ and $\widetilde{\alpha} \cap \tilde{\gamma} \neq \varnothing$.

If we show that $G$ is generated by the transformations $A_{\tilde{\alpha}}$, where $\alpha$ is a simple closed geodesic on $N$ and $\widetilde{\alpha} \cap \tilde{\gamma} \neq \varnothing$, then we can conclude that $\hat{g}$ commutes with every element of $G$. Let $x$ be a point on the geodesic $\gamma$ on 


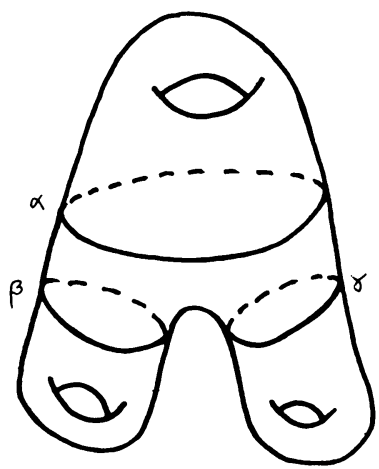

FIGURE 2

$N$. The above is equivalent to the assertion that $\Pi_{1}(N ; x)$ is generated by the simple loops based at $x$ that have a single transverse intersection with $\gamma$. From this point of view the result follows easily. In [4] Nielsen proves the two homeomorphisms inducing the same isomorphism on the fundamental group of a compact surface are homotopic. We conclude that $g$ is homotopic to the identity.

Another theorem of Nielsens [5, 7] asserts that if $f$ is a diffeomorphism whose $n$th iterate is homotopic to the identity, then $f$ is homotopic to a diffeomorphism $h$ so that the $n$th iterate of $h$ is the identity. We may conclude that $f$ is homotopic either to the identity or to an order two diffeomorphisms of $N$.

Let $\mu$ be a riemannian metric on $N$ and let $f^{*} \mu$ be the pull back of $\mu$ by $f$ to $N . \mu+f^{*} \mu$ defines a new metric on $N$ with respect to which $f$ is an isometry. It follows that $f$ is a conformal map in the complex analytic structure on $N$ induced by the new metric.

Proof of Theorem 2. As a consequence of the lemma we may suppose that $N$ has a complex analytic structure with respect to which $f$ is conformal. Let $S$ be a surface with boundary that is homeomorphic to the sphere with three disjoint open discs deleted. If $N$ has genus $p>2$ then there is an embedding $i: S \rightarrow N$ so that the boundary curves of $i(B)$ are disjoint closed geodesics $\alpha, \beta$, and $\gamma$, and $\alpha$ is a dividing curve on $N$. (See Figure 2.) The geodesics $\alpha$ and $\beta$ have a unique common perpendicular $\delta$ in $S$. Since $\alpha$ and $\beta$ are $f$-invariant and $\alpha$ is dividing we get $f(S)=S$ and $f(\delta)=\delta$. Then $f$ must fix $\delta$ pointwise and so $f$ is the identity.

If $N$ has genus two then we can choose three disjoint simple closed geodesics $\alpha, \beta$, and $\gamma$ where $\alpha$ is a dividing curve on $N$. As above $f$ is either the identity or when $f$ is restricted to either of the two subsurfaces $N-\{\alpha, \beta, \gamma\}$, $f$ is the involution of Lemma 1 . In the latter case we have seen that $f$ is the hyperelliptic involution.

Let $N^{\prime}$ denote the surface $N$ with a different complex analytic structure and let $g$ be the hyperelliptic involution of $N^{\prime}$. By Theorem $1, f$ and $g$ have the 
same action on free homotopy classes of simple closed curves. It follows as in the proof of Lemma 2 that $f \circ g^{-1}$ is homotopic to the identity, and therefore that $f$ is homotopic to the hyperelliptic involution for any complex analytic structure on $N$.

Let $N$ be a compact Riemann surface of genus $p>1$. The Teichmüller space $T(N)$ is the set of equivalence classes of pairs $\left(N_{0}, f_{0}\right)$ where $N_{0}$ is a Riemann surface and $f_{0}: N \rightarrow N_{0}$ is a homeomorphism. Two pairs $\left(N_{1}, f_{1}\right)$ and $\left(N_{2}, f_{2}\right)$ are equivalent if and only if there is a conformal map $h: N_{1} \rightarrow N_{2}$ so that $f_{2}^{-1} \circ h \circ f_{1}$ is homotopic to the identity. A homeomorphism $f: N \rightarrow N$ induces a map $f^{*}: T(N) \rightarrow T(N)$ by $f^{*}\left[N_{1}, f_{1}\right]=\left[N_{1}, f_{1} \circ f\right]$. The map $f^{*}$ depends only on the homotopy class of $f$ and thus describes an action of the mapping class group of the surface $N$ on $T(N)$.

Theorem 3. Let $f: N \rightarrow N$ be a homeomorphism of the compact surface $N$ of genus $p>1$. Then $f$ acts trivially on the Teichmüller space $T(N)$ if and only if, for every simple closed geodesic $\alpha$ on $N, f(\alpha)$ is freely homotopic to either $\alpha$ or $-\alpha$.

Proof. Suppose that $f(\alpha)$ is freely homotopic to $\alpha$ or $-\alpha$ for all simple closed geodesics $\alpha$ on $N$. By Lemma 2 either $f$ is homotopic to the identity, in which case it clearly acts trivially on $T(N)$, or $N$ is a surface of genus two and $f$ is homotopic to the hyperelliptic involution. We must consider the later case.

Given a Riemann surface $N_{1}$ of genus two and a homeomorphism $f_{1}: N \rightarrow$ $N_{1}$ observe that the map $f_{1} \circ f \circ f_{1}^{-1}: N_{1} \rightarrow N_{1}$ sends every simple closed geodesic $\alpha$ to a curve that is freely homotopic to $\alpha$ or $-\alpha$. Applying the lemma again we conclude that $f_{1} \circ f \circ f_{1}^{-1}$ is homotopic to the hyperelliptic involution. It follows that $f$ acts trivially on $T(N)$.

Conversely suppose that for some simple closed geodesic $\alpha$ on $N, f(\alpha)$ is not freely homotopic to one of $\alpha$ or $-\alpha$. Let $\beta$ denote the geodesic on $N$ freely homotoopic to $f(\alpha)$. Using standard methods in hyperbolic geometry [1] it can be shown that there is a surface $N_{1}$ and a map $f_{1}: N \rightarrow N_{1}$ so that the geodesics on $N_{1}$ freely homotopic to $f_{1}(\alpha)$ and $f_{1}(\beta)$ have different lengths. Basically the idea is that if $\alpha \cap \beta=\varnothing$ then the lengths can be varied independently by a deformation of the surface. If $\alpha \cap \beta \neq \varnothing$ then by a deformation the length of $\alpha$ can be made arbitrarily small, forcing the length of $\beta$ to get large. It is therefore impossible to have a conformal map $h: N_{1} \rightarrow N_{1}$ which is homotopic to $f_{1} \circ f \circ f_{1}^{-1}$ since it would have to identify geodesics of different lengths. Thus $f$ does not act effectively on $T(N)$.

In conclusion we remark that with minor modification the results are true for Riemann surfaces with distinguished points; in other words, punctures, holes, or ramification points. Lemma 1 extends to the case where $S$ is a sphere with three distinguished points, two of which are of the same geometric type. See [3] for a complete statement and proof. The class of surfaces constructed by gluing together pairs of these generalized three-holed spheres (along with some 
simpler surfaces) takes the place of the surface of genus two in the general case. This provides an alternative approach to Theorem 1 in Singerman's paper [6].

\section{REFERENCES}

1. W. Abikoff, The real analytic theory of Teichmüller space, Lecture Notes in Math., vol. 820, Springer-Verlag, New York, 1980.

2. H. Farkas and I. Kra, Riemann surfaces, Springer-Verlag, New York, 1980.

3. A. Haas, Diophantine approximation on hyperbolic orbifolds, Duke J. Math. 56 (1988), 531547.

4. J. Nielsen, Untersuchungen Zur Topologie der geschlossenen zweiseitigen Flächen, Acta Math. 50 (1927), 189-358.

5. J. Nielsen, Abbildungsklassen endlicher Ordnung, Acta Math. 75 (1942), 23-115.

6. D. Singerman, Finitely maximal Fuchsian groups, J. London Math. Soc. (2) 6 (1972), 29-38.

7. H. Zieschang, E. Vogt, and H. D. Coldewey, Surfaces and planar discontinuous groups, Lecture Notes in Math., vol. 835, Springer-Verlag, New York, 1980.

\begin{tabular}{|c|c|c|c|c|c|c|c|}
\hline $\begin{array}{l}\text { DEPARTMENT } \\
\text { CTICUT } 06268\end{array}$ & oF & Mathematics, & UNIVERSITY & CONNE & IICUT, & STORRS, & CON- \\
\hline $\begin{array}{l}\text { DEPARTMENT } \\
\text { CTICUT } 06320\end{array}$ & OF & Mathematics, & CONNECTICUT & College, & NeW & LONDON, & CON- \\
\hline
\end{tabular}

